\title{
Analysis on Operation Mode of Y-Type Rail Transit in Suburbs
}

\author{
Qiqi $\mathrm{GAO}^{\mathrm{a}}$, Baohua $\mathrm{MAO}^{\mathrm{a}, 1}$, Min $\mathrm{WANG}^{\mathrm{b}}$, Qi ZHOU ${ }^{\mathrm{b}}$ and $\mathrm{Yi} \mathrm{GE}^{\mathrm{c}}$ \\ ${ }^{a}$ Key Laboratory of Transport Industry of Big Data Application Technologies for \\ Comprehensive Transport, Beijing Jiaotong University Beijing, China \\ ${ }^{\mathrm{b}}$ School of Transportation Beijing Jiaotong University Beijing, China \\ ${ }^{\mathrm{c} C h i n a ~ R a i l w a y}$ Siyuan Survey and Design Group Co., LTD, China
}

\begin{abstract}
The construction of a Y-type urban rail transit line can effectively solve the problems of low line coverage and low utilization in suburban areas. The purpose of this paper is to study and analyze the effect of different operation modes of the Y-type line of suburban urban rail transit. In this paper, the objective function is to minimize value of passenger travel time costs and enterprise operating costs, and the main and branch train frequencies are used as decision variables to establish a selection model for Y-type operation mode, and design an improved multi-objective optimization algorithm based on NSGA-II. A case study was carried out on the Y-type line to evaluate the effect of each scheme. The results of the study found that there is no obvious difference between the advantages and disadvantages of the three operation modes. The passenger waiting time under the independent operation plan is the highest but the comfort level is greater, and the train travel distance is the lowest. The partial- through operation accommodates the interests of both passenger and business. Therefore, the corresponding operating mode should be selected according to the preferences of passengers and enterprises.
\end{abstract}

Keywords. Operation mode, Y-type line, NSGA-II

\section{Introduction}

In recent years, the scale of urbanization in China continues to expand, and the large number of population brings together to increase the travel demand, and the traffic travel problems follow. Urban rail transit can effectively connect the central area and the suburbs, solve the problems of passenger travel and urban traffic congestion, and become the general trend of the development of urban transportation in the future. With the continuous expansion of the rail transit line network, the line forms are also changing constantly, and $\mathrm{Y}, \mathrm{X}$ and $\mathrm{U}$ lines appear one after another. Among them, Y - shaped line is widely used at home and abroad [1].

At present, domestic and foreign scholars have carried out extensive research on Y- type line. $\mathrm{Li} \mathrm{[2]} \mathrm{analyzed} \mathrm{various} \mathrm{typical} \mathrm{road} \mathrm{features} \mathrm{of} \mathrm{urban} \mathrm{rail} \mathrm{transit,} \mathrm{providing}$ a basis for the selection of Y-shaped line operation mode. Zhu [3] respectively took a single line and the whole road network as the research object to establish the optimization

${ }^{1}$ Corresponding Author, Baohua MAO; Key Laboratory of Transport Industry of Big Data Application Technologies for Comprehensive Transport, Beijing Jiaotong University, Beijing 10044, China. E-mail: bhmao@bjtu.edu.cn. 
model of operation scheme and put forward the optimization method. In view of the shortage of station supply, Canca D puts forward an operation mode - adding small transit trains in large passenger flow section [4]. Yan [5] analyzed the centripetal characteristics and sectional characteristics of passenger flow on Y-type lines of urban rail transit on this basis. Zhang [6] analyzed the centripetal characteristics and sectional characteristics of passenger flow on Y-shaped lines. Liu [7] established an optimization model with passenger travel time and train travel kilometers as objective functions. Ge [8] constructed an appropriate Y-type route model according to the characteristics of passenger flow, built the model with passenger satisfaction and enterprise operating cost as objective functions, and solved the optimal route scheme under different weight coefficients and carried out comparative analysis. Shi [9] established an optimization model of multi-route train operation scheme with maximizing the utilization rate of line transmission capacity, minimizing the number of trains on line and passenger travel costs. Wang [10] established a line load level evaluation model including the two-dimensional index of load mean and standard margin. Mao [11] analyzed the characteristics of network operation technologies such as multi-route, multi-group, fast and slow cars, and over-track operation, and proposed the applicability of different methods.

In this paper, a selection model for Y-type line operation mode is established, and the objective function is to minimize the passenger travel cost and enterprise operation cost, and the passenger flow of a city is taken as the calculation data to formulate the Y-type line operation plan. And the operation effect is analyzed through various indicators such as passenger travel time and line load factor.

\section{Selection Model for Y-Type Operation Mode}

The operation effect of different operation modes of Y-type lines is quite different. The Y-shaped line shown in figure 1 will be taken as the research object in this paper $(1<\mathrm{x}<\mathrm{k}<\mathrm{m}<\mathrm{n})$, the operation effect under different operation routes is calculated, and the operation effect is analyzed by indicators such as passenger waiting time, train travel distance, load factor, etc. As can be seen from the figure, the main line is $[1, \mathrm{~m}]$, and one route is opened. The branch line is $[\mathrm{k}, \mathrm{n}]$, and the branch line will turn back at station $\mathrm{x}$. The upward direction is from 1 to $\mathrm{m}$ for mainline trains and from $\mathrm{x}$ to $\mathrm{n}$ for regional trains.

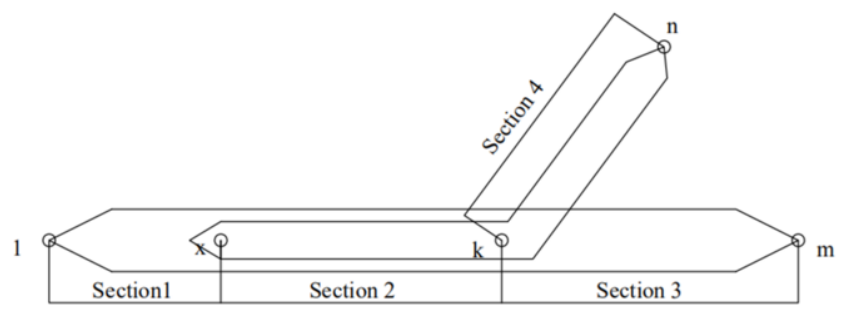

Figure 1. Schematic diagram of the research object line structure. 


\subsection{Model Assumptions}

The actual operation situation is relatively complex, and the operation effect evaluation is mainly conducted with the interests of passengers and enterprises as the target. Therefore, it is necessary to make assumptions about the train operation process and passengers' departure behavior:

(1) The train marshalling scheme is fixed and the running speed unchanged;

(2) All trains stop at each station;

(3) The arrival of passengers is considered to be uniformly distributed;

(4) Passengers will choose the direct route firstly;

(5) The use of vehicle undersides on main and branch lines is independent;

(6) Passengers in the collinear section shall be shared in proportion to the running frequency of the main and branch lines.

\subsection{Variable Symbol and Definition}

All variables and their meanings in the model are shown in the following table.

Table 1. Variable symbols and their definitions in the model.

\begin{tabular}{|c|c|}
\hline Variable & definition \\
\hline$C$ & Vehicle capacity \\
\hline $\boldsymbol{m}$ & Number of main line stations \\
\hline $\boldsymbol{n}$ & Total number of main and branch line stations \\
\hline$d$ & $d=1$ represents upward, $d=2$ represents downward \\
\hline $\boldsymbol{h}$ & Road marking, $h=1$ represents main line route, $h=2$ represents branch line route \\
\hline$f_{h}$ & The train operation frequency of route $h$ \\
\hline$f_{0}$ & The minimum train operation frequency \\
\hline $\boldsymbol{f}_{m}$ & The maximum capacity of line \\
\hline $\boldsymbol{b}$ & Section; $b=1,2,3,4$ \\
\hline$c_{p q}$ & passenger flow volume from $\mathrm{p}$ to $\mathrm{q} ; p, q \in b$ \\
\hline$P_{g}^{d}$ & $\begin{array}{l}\text { Different kinds of passenger flow, division according to the beginning and end section; } \\
\qquad g=1,2,3,4\end{array}$ \\
\hline$\lambda_{h P_{g}^{d}}$ & The sharing rate of passenger flow in route $h$ \\
\hline$S_{h P_{g}^{d}}$ & Whether route $h$ undertakes passenger flow $P_{g}^{d}, 1$ means yes, else 0 \\
\hline$t_{w}^{1}, t_{w}^{2}$ & Passenger waiting time \\
\hline $\boldsymbol{Z}$ & Objective function \\
\hline$\omega$ & Weight coefficient \\
\hline$\alpha$ & Design full load rate \\
\hline$\alpha_{\max }$ & Maximum section load factor \\
\hline $\bar{\alpha}$ & Average load factor of line \\
\hline$L_{h, d}$ & The length of path $h$ in the direction $d, \mathrm{~km}$ \\
\hline$r$ & The interval between adjacent stations \\
\hline$q_{h, r}$ & Passenger flow shared by the train with route $h$ in interval $r$ \\
\hline$N$ & Number of train formation vehicles, 6 \\
\hline $\boldsymbol{\beta}_{h}^{r}$ & Whether $\mathrm{h}$ covers the interval $\mathrm{r} ; 1$ means yes, else 0 \\
\hline
\end{tabular}

\subsection{The Objective Function}

The train operation of urban rail transit mainly involves two objects: passengers and operating enterprises. Passengers want journeys as short as possible; Operating enterprises hope to consume as little cost as possible [12]. The interests of both parties 
are mutually restricted and contradictory, so the model should take into account the interests of both passengers and operating enterprises.

Firstly, the FAHP is used to determine the main influencing factors and their weights. The model is established with the goal of minimizing passenger travel time and train travel distance finally.

(1)Passenger travel time

The passenger waiting time is minimized to represent the minimization of passenger travel time cost.

First of all, passenger flow on Y-shaped line can be classified according to the operating frequency of passenger trains, as shown in table 2.

Table 2. Passenger flow classification on Y-type line.

\begin{tabular}{cccc}
\hline $\begin{array}{c}\text { Kinds of passenger } \\
\text { flow }\end{array}$ & $\begin{array}{c}\text { Beginning } \\
\text { section }\end{array}$ & $\begin{array}{c}\text { End } \\
\text { section }\end{array}$ & $\begin{array}{c}\text { Train operation } \\
\text { frequency }\end{array}$ \\
\hline 1 & 1 & 1 & $f_{1}$ \\
& 1 & 2 & $f_{1}$ \\
& 1 & 3 & $f_{1}$ \\
& 2 & 3 & $f_{1}$ \\
& 3 & 3 & $f_{2}$ \\
2 & 2 & 4 & $f_{2}$ \\
3 & 4 & 4 & $f_{1}+f_{2}$ \\
4 & 3 & 3 & $f_{1} \& f_{2}$ \\
\hline
\end{tabular}

As can be seen from table 2, there are four types of passenger flows on Y-type route: $\mathrm{P}_{1}$ : Only take the train on the main line route, $P_{1}^{1}=c_{11}+c_{12}+c_{13}+c_{23}+c_{33}$.

$\mathrm{P}_{2}$ : Only take the train on the branch line route, $P_{2}^{1}=c_{24}+c_{44}$.

$\mathrm{P}_{3}$ : Main line and branch line are optional, $P_{3}^{1}=c_{22}$.

$\mathrm{P}_{4}$ : Take both the main and branch interchanges, $P_{4}^{1}=c_{14}$.

Then, the share rate of $h$ in the upward direction to various passenger flows is:

$$
\lambda_{h P_{g}^{1}}=S_{h P_{g}^{1}} \cdot \frac{f_{h}}{S_{1 P_{g}^{1}} \cdot f_{1}+S_{2 P_{g}^{1}} \cdot f_{2}}
$$

Considering the uniform distribution of passenger arrivals, the waiting time of passengers can take half of the train departure interval, and the waiting time of passengers upward is:

$$
t_{w}^{1}=\sum_{h=1}^{2} \sum_{g=1}^{4}\left(\lambda_{h P_{g}^{1}} \cdot P_{g}^{1} / 2 f_{h}\right)
$$

The waiting time of passengers downward is:

$$
t_{w}^{2}=\sum_{h=1}^{2} \sum_{g=1}^{4}\left(\lambda_{h P_{g}^{2}} \cdot P_{g}^{2} / 2 f_{h}\right)
$$

The minimum objective function of passenger waiting time is: 


$$
\min Z_{1}=t_{w}^{1}+t_{w}^{2}
$$

(2) Enterprise operating cost

The train travel distance is chosen to represent the operating cost of the enterprise:

$$
\min Z_{2}=\sum_{h=1}^{2} \sum_{d=1}^{2} L_{h, d} \cdot f_{h}
$$

(3) The multi-objective function is transformed into a single objective function

The model is a multi-objective function, and the two objectives are mutually opposite, the simultaneous optimization of the two objectives cannot be achieved. So linear weighting method is adopted to carry out multi-objective transformation..

Due to the different units and orders of magnitude of the two targets, linear weighting method will be used after dimensionless. The dimensionless formula is as follows:

$$
\overline{Z_{\mu}}=\frac{Z_{\mu}-\min Z_{\mu}}{\max Z_{\mu}-\min Z_{\mu}}
$$

The single objective function is as follows:

$$
\min Z=\varphi_{1} \times \overline{Z_{1}}+\varphi_{2} \times \overline{Z_{2}}
$$

According to the results of the fuzzy analytic hierarchy process, the weights of $Z_{1}, Z_{2}$ are 0.2415 and 0.27 respectively, which are normalized and can be obtained $\varphi_{1}=0.472$, $\varphi_{1}=0.528$.

\subsection{Constraint conditions}

(1) Minimum departure frequency of independent operation:

$$
f_{h} \geq \max _{r \in R_{h}}\left\{\frac{\sum r q_{h, r} \cdot L_{h, r, d}}{C \cdot N \cdot \alpha \cdot L_{h, d}}, f_{0}\right\}(h=1,2)
$$

(2) Minimum departure frequency of collinear operation:

$$
f_{1}+f_{2} \geq \max _{r \in R_{h}}\left\{\frac{\sum_{r} q_{h, r} \cdot L_{h, r, d}}{C \cdot N \cdot \alpha \cdot L_{h, d}}, f_{0}\right\}
$$

(3) Maximum capacity of line:

$$
f_{1}+f_{2} \leq f_{m}
$$




\subsection{Evaluation Index}

(1) Passenger waiting time

$$
\begin{aligned}
t_{w}^{1} & =\sum_{h=1}^{2} \sum_{g=1}^{4}\left(\lambda_{h P_{g}^{1}} \cdot \frac{P_{g}^{1}}{2 f_{h}}\right) \\
t_{w}^{2} & =\sum_{h=1}^{2} \sum_{g=1}^{4}\left(\lambda_{h P_{g}^{2}} \cdot \frac{P_{g}^{2}}{2 f_{h}}\right)
\end{aligned}
$$

(2) Train travel distance

$$
\min Z_{2}=\sum_{h=1}^{2} \sum_{d=1}^{2} L_{h, d} \cdot f_{h}
$$

(3) Maximum load factor

$$
\alpha_{\max }=\max \sum_{h=1}^{2} \frac{\beta_{h}^{r} \cdot q_{h, r}}{f_{h} \cdot N \cdot C}
$$

(4) Average load factor of line

$$
\bar{\alpha}=\frac{1}{n-1} \sum_{r=1}^{n-1} \sum_{h=1}^{2} \frac{\beta_{h}^{r} \cdot q_{h, r}}{f_{h} \cdot N \cdot C}
$$

\section{Solution Algorithm}

This paper solves a multi - objective nonlinear optimization problem.

\subsection{Non-Dominant Sorting Genetic Algorithm}

At present, there are many multi-objective optimization algorithms, among which the most widely used is Kalyanmoy Deb's Non-Dominant Sorting Genetic Algorithm with Elite Strategy (NSGA-II) [13]. The algorithm used in this paper is Gamultiobj, which is a multi-objective optimization algorithm improved based on NSGA-II. The organizational structure of this algorithm is shown in figure 2 . 


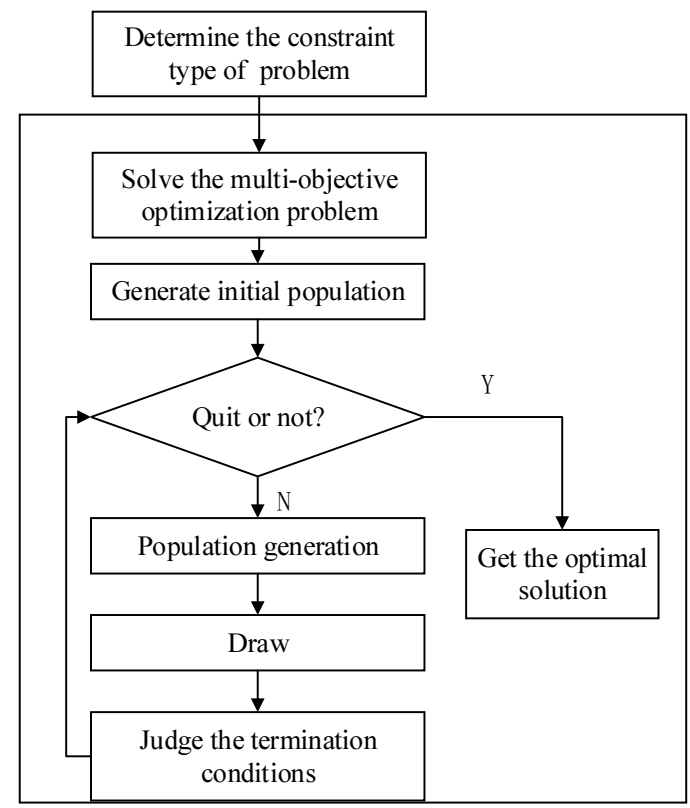

Figure 2. Organization chart of gamultiobj.

\subsection{Pareto Optimal Solution}

Pareto optimal solution is a state of optimal allocation of resources. For multi-objective function, there is no absolute optimal solution, and the result of multi-objective optimization can usually get a solution set, which is called the Pareto optimal set. The objective function corresponding to the decision vector in the Pareto optimal set is graphically represented, which is called the Pareto frontier.

\section{Model Calculation and Result Analysis}

\subsection{Case Introduction}

A city rail transit Y-type line is shown in figure 3. The line has 14 stations. Only stations $1,4,7,10$ and 14 have the conditions to turn back. Adjacent station intervals are numbered and marked as shown in the figure. The distance between line stations is shown in table 3. 


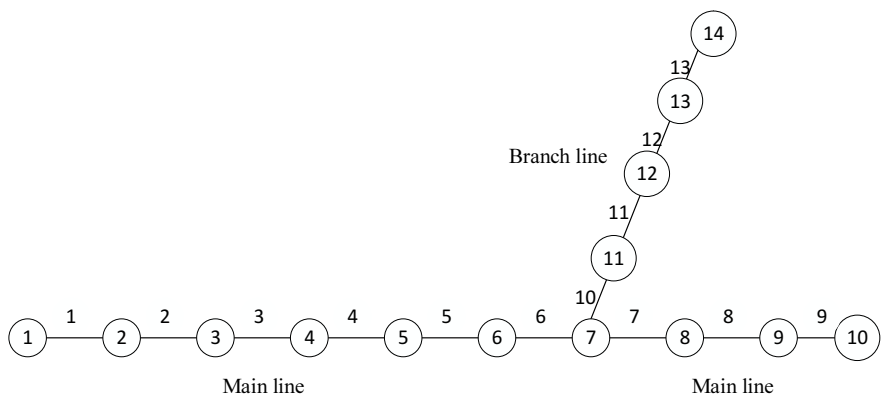

Figure 3. Schematic diagram of Y-type rail transit line in a certain city.

Table 3. Interval length of urban rail transit lines.

\begin{tabular}{cccccccccccccc}
\hline Interval & $\mathbf{1}$ & $\mathbf{2}$ & $\mathbf{3}$ & $\mathbf{4}$ & $\mathbf{5}$ & $\mathbf{6}$ & $\mathbf{7}$ & $\mathbf{8}$ & $\mathbf{9}$ & $\mathbf{1 0}$ & $\mathbf{1 1}$ & $\mathbf{1 2}$ & $\mathbf{1 3}$ \\
\hline Length $(\mathrm{km})$ & 2.5 & 2.3 & 2.2 & 1.8 & 1 & 1.3 & 1.2 & 1.3 & 1.5 & 1 & 1.2 & 1.3 & 1.5 \\
\hline
\end{tabular}

(1)Cross-section passenger flow of peak hour

Y-type lines are complex, and passenger flows of main and branch lines need to be calculated separately. The results are shown in table 4.

Table 4. Passenger flow of intervals between adjacent stations.

\begin{tabular}{cccccccccccccc}
\hline Interval & $\mathbf{1}$ & $\mathbf{2}$ & $\mathbf{3}$ & $\mathbf{4}$ & $\mathbf{5}$ & $\mathbf{6}$ & $\mathbf{7}$ & $\mathbf{8}$ & $\mathbf{9}$ & $\mathbf{1 0}$ & $\mathbf{1 1}$ & $\mathbf{1 2}$ & $\mathbf{1 3}$ \\
\hline Passenger & 66 & 132 & 199 & 272 & 308 & 301 & 117 & 70 & 26 & 133 & 89 & 51 & 20 \\
flow & 66 & 35 & 10 & 10 & 95 & 66 & 42 & 07 & 21 & 60 & 73 & 60 & 95 \\
\hline
\end{tabular}

(2)Parameter values. The results are shown in table 5.

Table 5. Model and algorithm parameter values.

\begin{tabular}{cccc}
\hline Parameter & Definition & Value & Unit \\
\hline $\boldsymbol{f}_{\boldsymbol{m}}$ & The maximum discharge capacity of line & 30 & pair/h \\
$\boldsymbol{f}_{\mathbf{0}}$ & The minimum train operation frequency & 6 & pair/h \\
$\mathrm{C}$ & Vehicle capacity & 247 & person \\
$\vartheta$ & Train return operation time & 2 & $\min$ \\
$\alpha$ & Design full load rate & 80 & $\%$ \\
\hline
\end{tabular}

\subsection{Case Solution}

The different positions of the starting point and return station of the Y-type branch road form different traffic schemes: The all-through operation is formed when the return station is the first; The partial-through operation is formed when the return station is the fourth; The independent operation is formed when the return station is the seventh.

The optimal Pareto solution and Pareto front under each scheme are as shown in figure 4 . 


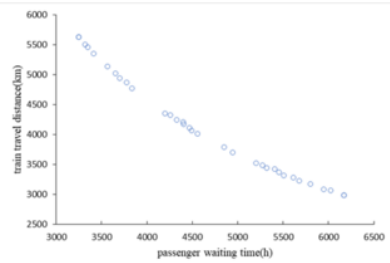

(a) Turn-back station is 1

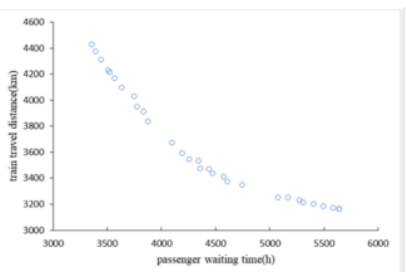

(b) Turn-back station is 4

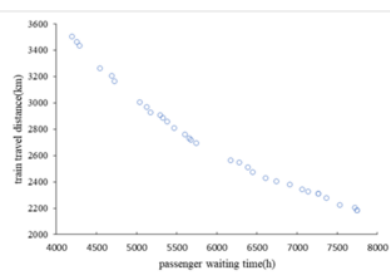

(c) Turn-back station is 7

Figure 4. Pareto front when the turn-back station is 1.

As can be seen from figure 4, the train travel distance decreases with the increase of passenger waiting time, and the rate of change decreases gradually. The optimal solutions of the three operation modes are summarized as follows:

Table 6. Data summary of the three operation modes.

\begin{tabular}{|c|c|c|c|c|}
\hline \multirow{2}{*}{ Operation mode } & \multicolumn{2}{|c|}{ Frequency (pair/h) } & \multirow{2}{*}{$\begin{array}{c}\text { Passenger waiting } \\
\text { time(h) }\end{array}$} & \multirow{2}{*}{$\begin{array}{c}\text { Train travel distance } \\
(\mathbf{k m})\end{array}$} \\
\hline & Trunk & Branch & & \\
\hline \multirow{2}{*}{$\begin{array}{l}\text { All- through operation } \\
\text { Partial- through } \\
\text { operation }\end{array}$} & 11 & 11 & 4563 & 4006 \\
\hline & 15 & 11 & 4362 & 3475 \\
\hline Independent operation & 16 & 10 & 5744 & 2692 \\
\hline
\end{tabular}

As can be seen from table 6, partial- through operation is a reasonable operation scheme, where passengers waiting time and train travel mileage are $4362 \mathrm{~h}$ and $3475 \mathrm{~km}$ respectively, balancing the interests of passengers and enterprise. For the benefit of both passengers and enterprises, the effect of all-through operation is at the medium level. Therefore, when comparing the operation effect, if more attention is paid to the travel experience of passengers, the partial- through operation will have a better effect. If more attention to the interests of the enterprise, the independent operation is better.

The load factor of the three operation schemes is shown in table 7.

Table 7. Load factor of line section under different schemes.

\begin{tabular}{cccccc}
\hline $\begin{array}{c}\text { Operation } \\
\text { modes }\end{array}$ & $\begin{array}{c}\text { Maximum } \\
\text { section load } \\
\text { factor (\%) }\end{array}$ & All-line & $\begin{array}{c}\text { Main line before } \\
\text { bifurcation }\end{array}$ & $\begin{array}{c}\text { Main line after } \\
\text { bifurcation }\end{array}$ & $\begin{array}{c}\text { Branc } \\
\text { h }\end{array}$ \\
\hline $\begin{array}{c}\text { All- through } \\
\text { operation } \\
\text { Partial- } \\
\text { through } \\
\text { operation }\end{array}$ & 94.8 & 54.3 & 65.5 & 43.7 & 45.4 \\
$\begin{array}{c}\text { Independent } \\
\text { operation }\end{array}$ & 130.3 & 53.3 & 68 & 34.3 & 45.4 \\
\hline
\end{tabular}

As can be seen from table 7, the section load factor of the independent operation This is because the only branch with independent operation does not cover the section with the largest passenger flow, resulting in the main line bearing most of the passenger flow.

For passengers, the load factor is related to the comfort of their passengers. Over load factor will increase the congestion degree in the carriage, reducing the passenger comfort, but improve the train efficiency. Based on this, we can see that under the allthrough operation, the section load factor is $94.8 \%$, which is very close to full load. For passengers, the congestion degree is not high, and for trains, the train utilization rate is 
high, so the operation effect is good.

From the perspective of average line load factor, the three operation schemes are ranked from high to low in the following order: independent operation, all-through operation, and partial- through operation. Because the passenger flow of the whole line is unevenly distributed, the average load factor is all lower than $70 \%$. The average load factor of the three operation schemes in the section after the main bifurcation is relatively low, less than $50 \%$. The comfort of passengers in this section is high, but the use efficiency of trains is low.

\section{Conclusion}

In this paper, the passenger waiting time and train travel distance are minimized as objective functions, and the selection model for Y-type operation mode is established with the main and branch train frequency as variables. The improved multi-objective optimization algorithm based on NSGA-II is used to solve the problem, getting the train running frequency of three optimal operating schemes, namely all-through operation, partial- through operation, independent operation, and the operating effect of different schemes is evaluated by the indexes of maximum section load factor and average section 1 load factor.

Among the three operation schemes, the independent operation scheme has the highest passenger waiting time and the lowest train travel distance. The waiting time of passengers and the train travel distance under partial-through operation scheme are relatively reasonable. All- through operations have no advantage in any of the three schemes. In the analysis of the average load factor, except for the section after the main line bifurcation, all the other sections have the high average load ratio of the independent operation scheme. In the section after the main line bifurcation and the branch line, the average section load factor has little difference, and the passenger travel comfort is guaranteed.

To sum up, when comparing the operation effects of different train operation schemes, the three schemes have no obvious advantages and disadvantages, and the final choices are different due to different concerns. In the actual case analysis, it is necessary to combine the actual data and focus, according to the preferences of passengers and enterprises can choose the corresponding scheme.

\section{Acknowledgments}

This paper was supported by research grants from the National Natural Science Foundation of China (No. 71621001) and National Natural Science Foundation of China (71971021).

\section{Reference}

[1] Liu Y 2019 Research on the preparation of the Y-type train routing plan of urban rail transit based on travel purpose [D]. Beijing: Beijing Jiaotong University. 
[2] Li J F and Wang B D 2009 Analysis of Typical Traffic Modes of Urban Rail Transit [J]. Railway Transport and Economy, 31(10): 54-58.

[3] Zhu Y T 2016 Study on optimization of urban rail transit train schedule considering passenger travel efficiency [D]. Beijing: Beijing Jiaotong University.

[4] Canca D, Barrena E and Laporte G 2016 A short-turning policy for the management of demand disruptions in rapid transit systems[J]. Annals of Operations Research, 246(1-2): 145-166.

[5] Zhang Y 2011 Research on Y-Type Line Node of Urban Rail Transit[J]. Urban Rapid Rail Transit, 24(1): 58-61

[6] Zhang Z Y 2016 Research on Y-type Operational Crossing of Urban Rail Transit [J]. Traffic and Transportation, 32(06): 19-21..

[7] LIU M 2017 Study on Urban Rail Transit Y-type Line Train Delivery Scheme [D]. Beijing: Beijing Jiaotong University.

[8] Ge Y 2019 Study on Urban Rail Transit Y-type Line Train Delivery Scheme [D]. Beijing Jiaotong University.

[9] Shi R J, Zheng M, Yao Z S and Lan Y J 2017 Optimization Method of Train Operation Scheme of Urban Rail Transit Considering the Utilization of Line Transmission Capacity [J]. Journal of Transportation Systems Engineering and Information Technology, 17(06): 214-220.

[10] Wang M, Mao B H, Yang Y Q, Shi R J, Wang Y Q 2021 Research on load level evaluation of urban rail transit line considering equilibrium [J]. Journal of Transportation Systems Engineering and Information Technology, 21(02):98-104+118.

[11] Mao B H, Zhang Z, Chen Z J, Jia W Z, He T J 2017 Review on Networked Operation Organization Technology of Urban Rail Trantransit [J]. Journal of Transportation Systems Engineering and Information Technology, 17(06):155-163.

[12] WEI Z W 2020 Particle Swarm Optimization Design of Y-type Rail Train Operation Scheme in Urban Mass Tranform [J]. Journal of Measurement Science and Instrumentation, 11(01):87-93.

[13] Xue X R and Guo J M 2013 Application of traditional genetic algorithm and improved NSGA-II algorithm in multi-objective optimization problems [J]. Boiler Technology, 44(06): 5-8. 